\title{
Avaliação da aceitação do game Motion Rehab por idosos com sequelas de AVE
}

\author{
Ana Carolina Bertoletti De Marchi ${ }^{1,2,3}$, Rafael Rieder ${ }^{1,3}$, Eliane Lucia Colussi ${ }^{2}$, \\ Magliani Reis Fiorin Martel ${ }^{2}$, Mateus Trombetta ${ }^{3,}$ Nathália Pinto Cechetti $^{1}$ \\ ${ }^{1}$ Programa de Pós-Graduação em Computação Aplicada - Universidade de Passo Fundo \\ (UPF) - BR 285 - Bairro São José - CEP: 99052-900 - Passo Fundo - RS - Brasil \\ ${ }^{2}$ Programa de Pós-Graduação em Envelhecimento Humano - Universidade de Passo \\ Fundo (UPF) \\ ${ }^{3}$ Curso de Ciência da Computação - Universidade de Passo Fundo (UPF) \\ \{carolina, rieder, colussi\}@upf.br, maglifiorin@yahoo.com.br, \\ mateus.trombetta@hotmail.com, nathalia.cechetti@gmail.com
}

\begin{abstract}
The aim of the research was to evaluate the acceptance of the Motion Rehab game in elderly patients with stroke during a rehabilitation process. Ten men and women participated of the experiment, aged of $67.7 \pm 7.1$ years, predominantly ischemic stroke and frequenters of a Physical Rehabilitation Center. For our approach, we used a questionnaire based on the Technology Acceptance Model (TAM). The results show that the women elderly tend to better acceptance the use of game as a rehabilitation tool. The evaluation also points out that the game is a motivating factor, and stimulate performing exercises during each physiotherapy session.
\end{abstract}

Keywords. Activities of daily living, Aging, Attention, Games, Stroke.

Resumo. O objetivo da pesquisa foi avaliar a aceitação do game Motion Rehab por idosos com sequelas de AVE que o utilizaram em um processo de reabilitação. Participaram 10 idosos de ambos os sexos, com idade média de 67,7 \pm 7,1 anos, com predominância do AVE isquêmico e frequentadores de um Centro de Reabilitação Física. Para a avaliação foi utilizado um questionário construído com base no Modelo de Aceitação de Tecnologia (Technology Acceptance Model - TAM). Os resultados mostram que as idosas têm uma tendência maior a aceitar o game como ferramenta auxiliar de reabilitação. A avaliação também aponta que o game é um elemento motivador, servindo de estímulo à realização dos exercícios durante cada sessão de fisioterapia.

Palavras-chave. Acidente Vascular Encefálico, Atenção, Atividades Cotidianas, Envelhecimento, Jogos.

\section{Introdução}

O Acidente Vascular Encefálico (AVE) é uma das patologias neurológicas cada vez mais recorrentes em adultos e idosos, sendo que quando não resulta em óbito, deixa 
sequelas persistentes por toda a vida. De acordo com a World Health Organization (2013), trata-se de uma doença decorrente de uma interrupção da circulação sanguínea cerebral, classificada pela presença de hemorragia (rompimento de vasos sanguíneos) ou por isquemia (bloqueio da artéria por um coágulo), resultando em lesão do tecido cerebral.

As sequelas motoras do AVE são caracterizadas a partir da sua extensão e região afetada [Langhorne, et al., 2011] e podem atingir membro superior, tronco e membro inferior [Coupar et al., 2012], prejudicando, entre outros aspectos, a mobilidade, o equilíbrio, a marcha e a coordenação motora [Barbosa, 2012]. A presença de tais alterações compromete a execução das atividades de vida diária com interferência direta na qualidade de vida, o que torna fundamental a inserção do paciente em um processo de reabilitação o mais breve possível.

Dentre os processos de reabilitação atualmente disponíveis, pesquisadores estão inserindo em seus protocolos os games [Kim et al., 2011; Pompeu et al., 2014 e Vogiatzaki e Krukowski, 2014], que se caracterizam como uma ferramenta complementar, motivadora e diferenciada para o tratamento terapêutico. No entanto, a prova de sua eficácia entre os idosos ainda necessita de maiores pesquisas [Molina et al., 2014], principalmente no que se refere a sua aceitação.

Estudos como o de Hanif et al. (2011) sobre a aceitação de tecnologias em processo de reabilitação, comprovam que o seu uso como complemento em tratamentos fisioterapêuticos apresenta resultados positivos aos pacientes. Os autores mencionam facilidade de uso, acessibilidade, baixo custo da tecnologia, incentivo ao aumento da intensidade dos exercícios e motivação, como fatores desencadeadores desta aceitação. Da mesma forma, Nawaz et al. (2015), em uma revisão que avaliou a aceitação do uso de tecnologia em processos de reabilitação com idosos, concluíram que os participantes da maioria dos estudos incluídos aprovaram a experiência e sentiram-se satisfeitos.

Adicionalmente, Liu et al. (2015) apontam que esta aceitação também ocorre pelos profissionais terapêuticos. Os resultados do estudo indicam que em centros de reabilitação onde o uso de tecnologia nos tratamentos não é obrigatório, os profissionais, quando incentivados a inclui-la, passam a aceitá-la percebendo-a como um facilitador no tratamento. No entanto, ainda existe a necessidade de uma maior variedade de jogos e da diversificação de treinamentos [Hanif, Niaz e Khan, 2011].

Diante deste contexto, este trabalho tem como objetivo avaliar a aceitação do game Motion Rehab por idosos com sequelas de AVE que o utilizaram em um processo de reabilitação. Na sequência, o artigo apresenta a metodologia, os resultados, a discussão e a conclusão sobre o experimento.

\section{Metodologia}

Participaram do estudo 10 idosos com sequelas de AVE, de ambos os sexos, que realizaram um programa de reabilitação física e cognitiva proposto por Martel (2015), com o uso do game Motion Rehab. A intervenção foi realizada em 15 sessões, com duração de 45 minutos cada, executadas individualmente, uma vez por semana, em um Centro Regional de Reabilitação Física do Noroeste do Estado do Rio Grande do Sul. 
O game Motion Rehab (registrado no INPI sob número BR 512014 001087-2) foi desenvolvido por Fiorin et al. (2014) com base na fisioterapia convencional. O game possui uma interface $2 \mathrm{D}$ e é composto por quatro fases divididas em exercícios de flexão e abdução de ombro; extensão de cotovelo; extensão e flexão de quadril e joelho (Figura 1). Para a interação com o game os idosos realizavam os exercícios de frente para uma TV de 32 polegadas conectada a um computador e ao sensor de movimento Kinect, localizado abaixo da TV.

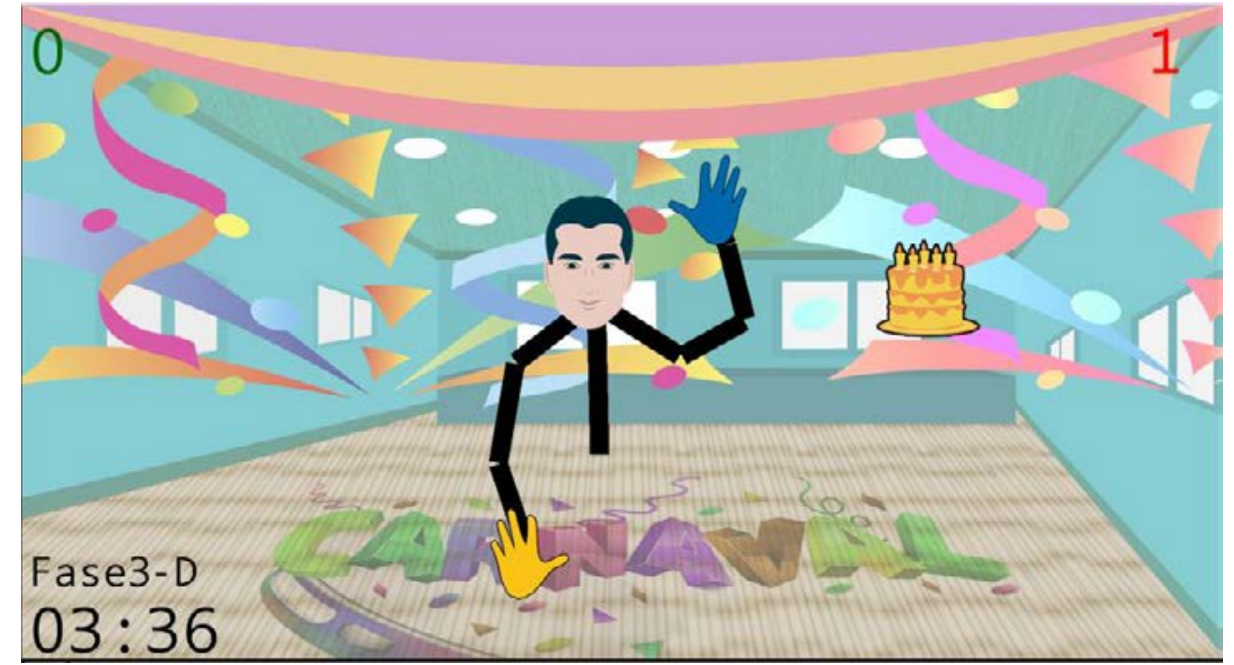

Figura 1. Fase 3 do Motion Rehab

Ao término do programa de intervenção, os idosos responderam ao questionário de avaliação de aceitação, construído com base no Modelo de Aceitação de Tecnologia (Technology Acceptance Model - TAM) proposto por Davis, Bagozzi e Warshaw (1989). O modelo é utilizado por pesquisadores para descrever a aceitação de tecnologias da informação após o seu uso [Dias et al., 2011]. A avaliação sustenta-se em três categorias: utilidade percebida, que determina o grau em que se acredita que o uso de uma tecnologia pode melhorar o desempenho e a produtividade; facilidade de uso, que corresponde ao grau em que se acredita que o uso do sistema de informação será livre de esforço, ou seja, fácil de aprender e interagir; e variáveis externas, que fornecem uma melhor compreensão do que influencia a utilidade percebida e a facilidade de uso.

O questionário utilizado conta com 16 questões agrupadas nas três categorias, conforme mostra a Tabela 1 . As respostas foram classificadas em uma escala de Likert de cinco pontos, sendo cinco o maior valor: concordo totalmente (5), concordo parcialmente (4), indiferente (3), discordo parcialmente (2) e discordo totalmente (1).

O estudo seguiu as Diretrizes e Normas Regulamentadoras de Pesquisas Envolvendo Seres Humanos, Resolução no 466/2012, e foi aprovado pelo Comitê de Ética em Pesquisa da Universidade de Passo Fundo sob parecer de ${ }^{\circ}$ 605.652/2014. Todos os participantes receberam informações acerca dos objetivos e procedimentos da pesquisa e assinaram o Termo de Consentimento Livre e Esclarecido.

Os dados foram tabulados e analisados estatisticamente no software SPSS 17. $\mathrm{Na}$ análise exploratória foram utilizadas as medidas de média e de desvio padrão (DP), 
e o teste Shapiro-Wilk (W) para avaliar a normalidade dos dados $(\alpha=0,05$, $W$ crítico $=0,842$ ).

Tabela 1. Questionário de avaliação da aceitação da tecnologia utilizado

\begin{tabular}{l|l}
\hline \multicolumn{2}{l}{ Categoria } \\
\hline ID & Questão \\
\hline \multicolumn{2}{l}{ Utilidade Percebida (U) } \\
\hline U1 & O Motion Rehab é importante e adiciona valor à minha reabilitação. \\
\hline U2 & O Motion Rehab é útil em minha reabilitação. \\
\hline U3 & O Motion Rehab torna minha reabilitação mais interessante. \\
\hline U4 & O Motion Rehab melhora meu desempenho na reabilitação. \\
\hline U5 & O Motion Rehab produz o efeito desejado em minha reabilitação \\
\hline U6 & O Motion Rehab aumenta a minha produtividade na reabilitação. \\
\hline U7 & O Motion Rehab motiva meu envolvimento na reabilitação. \\
\hline Facilidade de Uso (F) \\
\hline F1 & Usaria o Motion Rehab frequentemente se fosse fácil de utilizá-lo. \\
\hline F2 & Usaria o Motion Rehab frequentemente se tivesse o equipamento em casa. \\
\hline F3 & A interface do Motion Rehab facilita a realização da reabilitação. \\
\hline F4 & Usar o Motion Rehab é uma boa ideia. \\
\hline F5 & Usar o Motion Rehab é agradável. \\
\hline Variáveis Externas (V) \\
\hline V1 & Houve treinamento para usar o Motion Rehab. \\
\hline V2 & O fisioterapeuta tem bom nível de conhecimento sobre o Motion Rehab e ajudou-me a entendê-lo. \\
\hline V3 & Aprender a usar o Motion Rehab foi difícil pra mim. \\
\hline V4 & Frequentemente, confundo-me durante o uso do Motion Rehab. \\
\hline
\end{tabular}

Nas comparações entre grupos Masculino (GM) e Feminino (GF), utilizaram-se os testes t de Student (para distribuições normais) e Mann-Whitney (para distribuições não normais, $U$ crítico $=2$ ), $\operatorname{com} p<0,05$. A hipótese alternativa é de que a avaliação realizada pelos homens é diferente das mulheres.

\section{Resultados e Discussão}

A Tabela 2 apresenta os resultados da análise exploratória, e a Tabela 3 mostra os resultados da comparação entre grupos Masculino e Feminino. Já a Tabela 4 e a Tabela 5 consideram o agrupamento das respostas obtidas por categoria (Ugeral, Fgeral e Vgeral).

Com relação aos resultados da análise exploratória, nota-se na Tabela 2 e na Tabela 4 que a ampla maioria dos dados se apresenta com distribuição não normal. A exceção se faz para as amostras das questões U7 e F1, que estão normais.

Referente a significância estatística dos resultados, também observa-se na Tabela 3 e na Tabela 5 que somente dois itens apresentam relação significante (U2 e Ugeral). Em virtude do uso do teste Mann-Whitney para ambos os casos, é preciso também considerar o valor U para comprovar a significância.

Para U2, o valor U calculado é 2,5 e o U crítico é 2 para $p<0,05$. Logo, o resultado não é estatisticamente significativo. 
Já no caso de Ugeral, o valor U calculado é 391,5, e a distribuição é aproximadamente normal - o que confirma a significância do resultado. Nesta situação, pode-se dizer que existe diferença de percepção entre os grupos Masculino e Feminino para a categoria de avaliação "Utilidade Percebida". Considerando os dados da análise exploratória, relacionados a essa categoria, nota-se que as mulheres acham o uso do game Motion Rehab mais útil no processo de reabilitação do que os homens.

Tabela 2. Resultados da análise exploratória

\begin{tabular}{c|c|c}
\hline ID Questão & Média \pm DP & W calculado \\
\hline U1 & $4,5 \pm 0,527$ & 0,655 \\
\hline U2 & $4,4 \pm 0,516$ & 0,640 \\
\hline U3 & $4,2 \pm 0,632$ & 0,794 \\
\hline U4 & $4,1 \pm 0,876$ & 0,805 \\
\hline U5 & $4,2 \pm 0,632$ & 0,794 \\
\hline U6 & $3,9 \pm 0,876$ & 0,821 \\
\hline F1 & $3,9 \pm 0,994$ & $0,886^{*}$ \\
\hline F2 & $3,4 \pm 1,174$ & $0,873^{*}$ \\
\hline F3 & $4,1 \pm 0,483$ & 0,594 \\
\hline F4 & $4,7 \pm 0,483$ & 0,594 \\
\hline F5 & $4,5 \pm 0,527$ & 0,655 \\
\hline V1 & $4,5 \pm 0,527$ & 0,655 \\
\hline V2 & $4,6 \pm 0,516$ & 0,640 \\
\hline V3 & $2,2 \pm 0,789$ & 0,751 \\
\hline V4 & $2,6 \pm 1,265$ & 0,794
\end{tabular}

Tabela 3. Resultados da comparação entre grupos Masculino e Feminino

\begin{tabular}{c|c|c|c}
\hline ID Questão & GM - Média \pm DP & GF - Média \pm DP & p(value) \\
\hline U1 & $4,4 \pm 0,548$ & $4,6 \pm 0,548$ & 0,674 \\
\hline U2 & $4,0 \pm 0,000$ & $4,8 \pm 0,447$ & $0,048^{*}$ \\
\hline U3 & $4,0 \pm 0,707$ & $4,4 \pm 0,548$ & 0,465 \\
\hline U4 & $3,8 \pm 1,095$ & $4,4 \pm 0,548$ & 0,401 \\
\hline U5 & $3,8 \pm 0,447$ & $4,6 \pm 0,548$ & 0,095 \\
\hline U6 & $3,8 \pm 1,095$ & $4,0 \pm 0,707$ & 1,000 \\
\hline F1 & $3,6 \pm 1,140$ & $4,2 \pm 0,837$ & 0,374 \\
\hline F2 & $2,8 \pm 1,304$ & $4,0 \pm 0,707$ & 0,120 \\
\hline F3 & $4,4 \pm 0,548$ & $5,0 \pm 0,000$ & 0,144 \\
\hline F4 & $4,8 \pm 0,837$ & $4,4 \pm 0,548$ & 0,298 \\
\hline F5 & $4,6 \pm 0,548$ & $4,4 \pm 0,548$ & 0,674 \\
\hline V1 & $4,4 \pm 0,548$ & $4,6 \pm 0,548$ & 0,674 \\
\hline V2 & $4,6 \pm 0,548$ & $4,6 \pm 0,548$ & 0,920 \\
\hline V3 & $2,0 \pm 0,707$ & $2,4 \pm 0,894$ & 0,674 \\
\hline V4 & $2,2 \pm 1,095$ & $3,0 \pm 1,414$ & 0,401 \\
\hline
\end{tabular}


Tabela 4. Resultados da análise exploratória, agrupados por categoria

\begin{tabular}{c|c|c}
\hline ID & Média \pm DP & W calculado $>$ W crítico \\
\hline Ugeral & $4,171 \pm 0,742$ & $0,802>0,947$ \\
\hline Fgeral & $4,280 \pm 0,858$ & $0,747>0,947$ \\
\hline Vgeral & $3,475 \pm 1,358$ & $0,825>0,940$ \\
\hline
\end{tabular}

Tabela 5. Resultados da comparação entre grupos Masculino e Feminino, agrupados por categoria

\begin{tabular}{c|c|c|c}
\hline ID & GM - Média \pm DP & GF - Média \pm DP & p(value) \\
\hline Ugeral & $3,914 \pm 0,781$ & $4,429 \pm 0,608$ & $0,010^{*}$ \\
\hline Fgeral & $4,040 \pm 1,020$ & $4,520 \pm 0,586$ & 0,110 \\
\hline Vgeral & $3,300 \pm 1,418$ & $3,300 \pm 1,418$ & 0,435 \\
\hline
\end{tabular}

De maneira geral, analisando os resultados das três categorias de avaliação, percebe-se que, em média, o público feminino tem uma aceitação melhor do uso de tecnologias como ferramentas de intervenção auxiliares - o que era esperado, dado que as mulheres são mais abertas às novidades e mais engajadas em reabilitações diferenciadas. Tal fator também é percebido nos estudos de Kim et al. (2011) e Molina et al. (2014), por meio de uma proporção maior de mulheres nas amostras das pesquisas apontadas. Também nota-se que as mulheres apresentam, em média, um pouco mais de dificuldade para aprender a interagir com a ferramenta, e que, eventualmente, se confundem mais com os elementos virtuais do game do que os homens - também esperado, pois os homens tendem a ter melhor noção de espaço [Halpern, 2013].

Observa-se também que o público masculino, em média, sente que a intervenção é mais agradável com o uso do game. Isso pode demonstrar que os homens idosos tem interesse pela ferramenta, porém, precisam sentir mais segurança de movimentos com o game, para ter o mesmo resultado que imaginam ter com as ações cotidianas do fisioterapeuta.

Relacionado ao contexto do uso do game Motion Rehab observou-se inicialmente que os idosos tinham certa dificuldade na interação com o computador. Isso se justifica pois, para a grande parcela dos idosos, o momento da intervenção foi o primeiro contato com esta tecnologia.

Com o decorrer das sessões, também identificou-se que por meio da repetição semanal utilizando o game, os idosos obtiveram maior aceitação frente ao uso desta tecnologia. Durante a intervenção, era possível visualizar a motivação e o entusiasmo dos participantes, corroborando com os achados de Molina et al. (2014) e Hanif, Niaz e Khan (2011). Este último apontou alta aceitação do uso do game como uma ferramenta nova e interessante na reabilitação da deficiência motora.

Percebeu-se, ainda, que ao mesmo tempo em que eram exigidos movimentos corporais e manutenção de atenção, os estímulos que surgiam durante o jogo tornavam a sessão animada, sendo este um diferencial das sessões convencionais de reabilitação.

\section{Conclusão}

Este trabalho apresentou os resultados de uma avaliação de aceitação do game Motion Rehab por idosos com sequelas de AVE, realizada por profissionais da Saúde e da Computação. A análise estatística apontou, significativamente, que o grupo de pacientes 
idosas apresentou uma aceitação melhor ao game. Além disso, os observadores do experimento destacaram que, a cada sessão de reabilitação, o uso do game estimulava os pacientes a realizarem as atividades propostas pelo fisioterapeuta.

Adicionalmente, a partir dos resultados obtidos, uma nova versão do game foi desenvolvida para melhorar sua usabilidade. Na versão 2.0 foram implementadas melhorias quanto, por exemplo, acessibilidade, orientação e feedback. Além da versão 2.0, está sendo desenvolvida uma versão $3 \mathrm{D}$ do game com um cenário imersivo que explora a interação com elementos virtuais espalhados dentro do raio de alcance do paciente. Esta última versão tende motivar ainda mais os pacientes no processo de reabilitação, bem como dar suporte às atividades de domínio espacial.

Como trabalhos futuros, recomenda-se a aplicação do experimento com um grupo maior de pacientes junto ao Centro de Reabilitação Física, visando obter resultados mais significativos. Também sugere-se a disponibilização do game para os pacientes utilizarem em seu domicílio. Nesse caso, recomenda-se que uma nova versão do Motion Rehab tenha disponível um módulo para coleta e envio de dados pela web, para posterior análise e acompanhamento dos idosos em atividades de reabilitação.

\section{Agradecimentos}

Os autores agradecem ao CNPq, pelo apoio por meio do Edital MCTI/CNPQ/Universal 14/2014 e pela concessão da bolsa de Iniciação Científica, e à FAPERGS, pela concessão da bolsa de mestrado.

\section{Referências}

Barbosa, M. T. L. M. J. Custos e Efectividade da Reabilitação após Acidente Vascular Cerebral Uma Revisão Sistemática. 2012. 153 p. Dissertação (Mestrado em Gestão e Economia da Saúde) - Universidade de Coimbra, Coimbra.

Coupar, F.; Pollock, A.; Rowe, P.; Weir, C.; Langhorne, P. Predictors of upper limb recovery after stroke: a systematic review and meta-analysis. Clinical rehabilitation, v. 26, n. 4, p. 291-313, 2012.

Davis, F. D., Bagozzi, R. P., Warshaw, P. R. User Acceptance of computer technology: a comparison of two theoretical models. Manage Sci. New York. 1989; 35(8): 9821003.

Dias, G. A., Silva, P. M., Junior, J. B. D., Almeida, J. R. Technology Acceptance Model (TAM): avaliando a aceitação tecnológica do Open Journal Systems (OJS). Informação \& Sociedade: Estudos. 2011; 21(2):133-149.

Fiorin, M. R.; De Marchi, A. C. B. ; Rieder, R. ; Colussi, E. L.; Trombetta, M. Motion Rehab: um jogo sério para idosos com sequelas de Acidente Vascular Encefálico. In: XIV Workshop de Informática Médica, 2014, Brasília. Anais do CSBS. Brasília, 2014.

Halpern, D. F. Sex differences in cognitive abilities. Psychology press, 2013.

Hanif, M., Niaz, H., \& Khan, M. A. (2011, June). Investigating the possible role and usefulness of video capture virtual reality in motor impairment rehabilitation. In Next 
Generation Information Technology (ICNIT), 2011 The 2nd International Conference on (pp. 23-30). IEEE.

Kim, B. R., Chun, M. H., Kim, L. S., Park, Y. Effect of virtual reality on cognition in stroke patients. Annals of Rehabilitation Medicine, 2011; 35(4): 450-459.

Langhorne, P.; Bernhardt, J.; Kwakkel, Gert. Stroke rehabilitation. Lancet, v. 377, n. 9778, p. 1693-702, 2011. Elsevier.

Liu, L., Miguel Cruz, A., Rios Rincon, A., Buttar, V., Ranson, Q., \& Goertzen, D. (2015). What factors determine therapists' acceptance of new technologies for rehabilitation-a study using the Unified Theory of Acceptance and Use of Technology (UTAUT). Disability and rehabilitation, 37(5), 447-455.

Martel, M. R. F., Efeitos da intervenção com game na atenção e na independência funcional em idosos após Acidente Vascular Encefálico. Dissertação (Mestrado em Envelhecimento Humano) - Universidade de Passo Fundo, Passo Fundo, 2015.

Molina, K. I., Ricci, N. A., Moraes, A. S., Perracini, M. R. Virtual reality using games for improving physical functioning in older adults: a systematic review. Journal of NeuroEngineering and Rehabilitation, 2014; 11(156).

Moraes, E. N; Moraes, F. L., Lima, S. P. P. Características biológicas e psicológicas do envelhecimento. Revista Médica de Minas Gerais, Minas Gerais, v. 20, n. 1, p. 67$73,2010$.

Nawaz, A., Skjæret, N., Helbostad, J. L., Vereijken, B., Boulton, E., \& Svanaes, D. (2015). Usability and acceptability of balance exergames in older adults: A scoping review. Health informatics journal, 1460458215598638.

Pompeu, J. E., Alonso, T. H., Bordello, I. M., Alvarega, A. P. S. M., Torriani-Pasin, C. Os efeitos da realidade virtual na reabilitação do acidente vascular encefálico: Uma revisão sistemática. Motricidade, 2014; 10 (4): 111-122.

Vogiatzaki, E., Krukowski, A. Serious Games for Stroke Rehabilitation Employing Immersive User Interfaces in 3D Virtual Environment. Journal of Health Informatics. 2014; 6: 105-13.

World Health Organization. Stroke, Cerebrovascular accident: Health topics. Geneva, 2013. Disponível em: < http://www.who.int/topics/cerebrovascular_accident/en/>. Acesso em: 22 jun. 2013. 